\title{
DRAINAGE AFTER TOTAL KNEE REPLACEMENT
}

\author{
G. STERLING ${ }^{1}$, G. D. MCBAIN ${ }^{\square 2}$, J. A. HARRIS ${ }^{3}$ and M. BOLAND ${ }^{1}$
}

(Received 4 July, 2005)

\begin{abstract}
A simple lumped hydraulic model of knee drainage following arthroplasty is developed incorporating a pressure-volume equation of state for the knee capsule and a wound healing rate dynamically retarded by the blood flow-induced shear stress. The resulting secondorder nonlinear ordinary differential system is examined numerically and qualitatively to map the parameter space. In the model, moderate suction or a slight back-pressure promotes gradual drainage and healing whereas excessive suction can lead to a bifurcation in which healing is retarded or even prevented. Guided, then, by the model, the literature, and experience, continuous drainage with a small constant back-pressure appeared beneficial so we prospectively evaluated a series of ten patients. The results are consistent with the model and promising.
\end{abstract}

2000 Mathematics subject classification: primary 92C50; secondary $76 \mathrm{Z} 05$.

Keywords and phrases: wound bleeding, wound healing, blood-flow shear-retarded healing, nonlinear ordinary diffrential system, phase plane analysis.

\section{Introduction}

Suction drainage of wounds after total knee arthroplasty is used routinely by most orthopædic surgeons in the hope of avoiding the formation of postoperative hæmatoma, with the subsequent complications of infection and wound breakdown. Recently, this technique has been questioned in the literature, with some authors suggesting that suction drainage may not be necessary $[1,2,17,19]$. Most evidence, however, supports the use of drains, as long as they are removed within twenty-four hours $[6,11,12,16,22]$.

The best method by which to apply suction to the postoperative knee is unknown. Two questions remain -at what pressure, and by which means (continuous or intermit-

\footnotetext{
${ }^{1}$ Department of Orthopædics, Townsville General Hospital, Townsville, QLD, Australia.

${ }^{2}$ School of Aerospace, Mechanical, and Mechatronic Engineering, The University of Sydney, NSW, Australia.

${ }^{3}$ Maunsell Australia Pty Ltd, 21 Stokes St, Townsville QLD, Australia.

(C) Australian Mathematical Society 2006, Serial-fee code 1446-1811/06
} 


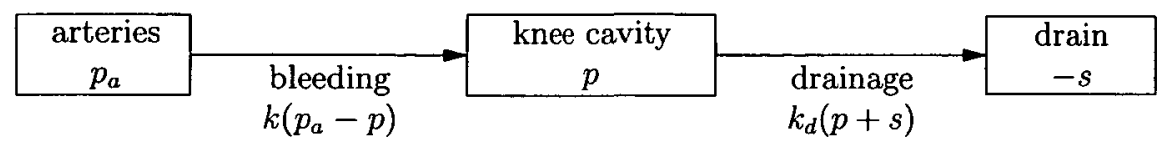

FIGURE 1. Three-reservoir hydraulic model of the draining knee cavity; each reservoir has a pressure, and the flow along the connecting branches is the product of a hydraulic conductance and the pressure drop.

tent) should drains be applied? Most surgeons use a spring-loaded (intermittent) drain, which provides a relatively high negative pressure. This device was designed for use in general surgery. Little is written in the literature to guide the orthopædic surgeon, but some evidence supports the use of a low-suction continuous device [3, 5, 14]. Berman et al., in a randomised prospective trial investigating drainage following orthopædic surgical wounds, found a clear advantage to using a continuous suction device over an intermittent device with respect to hæmatoma evacuation, wound drainage, wound healing, and possible complications [5]. Notably, knee arthroplasty patients receiving continuous suction experienced a greater drainage volume and better wound healing. How much suction to apply to the postoperative knee is also poorly understood, but some evidence suggests that low suction is a better alternative, with high-pressure drainage being associated with higher rates of tissue damage [3].

In this paper, in Section 2, we propose a simple lumped hydraulic model to explain drainage after total knee replacement, designed to be the simplest such model capable of producing the qualitative dynamics observed in actual postoperative patients. Mathematically the model consists of a low-order nonlinear ordinary differential system, and numerical solutions and qualitative analysis techniques are applied to map the parameter space and search for beneficial treatment strategies. In Section 3 we report how, guided by the model, we prospectively evaluated a series of primary cemented total knee replacement patients using a continuous drain with a small constant positive back-pressure. As discussed in Section 4, the measurements are qualitatively consistent with the model, and the two are also in unexpectedly good quantitative agreement.

\section{Total knee replacement drainage model}

The postoperative knee can be considered simply as three blood reservoirs that are arranged in a linear chain: arteries-knee-drain; see Figure 1. Each reservoir is associated with a pressure, and flow from one reservoir to the next is a function of the pressure difference between them. The arterial pressure is denoted by $p_{a}$, and the pressure in the knee cavity by $p$. The suction pressure in the external drain is denoted by $s$; a positive value of $s$ represents suction (that is, pressure less than atmospheric), whereas a negative value of $s$ represents a back-pressure. 


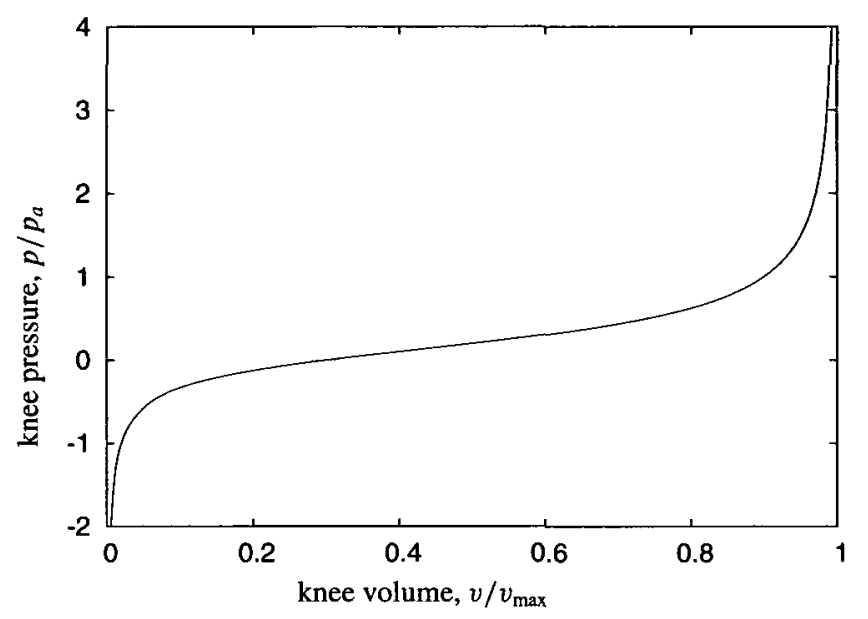

FIGURE 2. Pressure-volume relation for a knee, with $\alpha=0.5$ and $v_{0}=0.3 v_{\max }$ in (2.2). Representative values for the scales are maximum volume $v_{\max }=130 \mathrm{~mL}$, and arterial pressure $p_{a}=40 \mathrm{mmHg}$.

2.1. Mathematical model The time rate of change of the knee joint capsule volume, $\dot{v}$, is the difference between the rate of bleeding from the arterioles (and cancellous bone) into the knee and the rate of drainage from the knee into the drain:

$$
\dot{v}=\left\{k\left(p_{a}-p\right)\right\}-\left\{k_{d}(p+s)\right\} .
$$

Each flow is expressed as the product of a pressure difference and a hydraulic conductance (which may depend on the pressure difference and other system parameters). In the present model, $p_{a}, s$, and $k_{d}$ are considered constants; however, the knee pressure depends on the volume of fluid in the knee via an equation of state (Subsection 2.1.1) and the wound conductance $k$ changes with time as the wound heals (Subsection 2.1.2).

2.1.1. Pressure and volume in the knee The knee pressure $p$ is a monotonically increasing function of the knee volume $v$. Here the pressure-volume relation is postulated to be

$$
\frac{p}{p_{a}}=\alpha \frac{v-v_{0}}{\sqrt{v\left(v_{\max }-v\right)}}
$$

where $\alpha$ is a dimensionless constant with typical value 0.5 . This relationship is depicted in Figure 2 . As the knee volume approaches zero, the pressure tends towards $-\infty$; likewise, as the volume approaches its maximum $\left(v_{\max }\right)$, the pressure increases without bound. This behaviour is in keeping with experimental determinations of knee pressure-volume relationships $[8,21]$. The constants $v_{0}$ and $v_{\max }$ are the volumes at atmospheric and infinite pressure, respectively; $v_{\max }=130 \mathrm{~mL}$ and $v_{0} / v_{\max }=0.3$ seem to be representative. 
2.1.2. Healing: the rate of change of wound conductance The wound hydraulic conductance $k$ in (2.1) changes over time as the wound heals. We postulate that the healing rate should be:

- a maximum when there is no pressure difference or bleeding flow between the arteries and the knee capsule;

- a decreasing function of the absolute value of the pressure difference between the arteries and the knee, the flow rate between the arteries and the knee, or some combination of these, such as the shear stress applied to the wound surfaces by the flow; and

- zero when the conductance is zero (otherwise $k$ would become negative which is not physical as it implies fluid flow from lower to higher pressure).

Here it is assumed that the linear dimension characterising the wound opening decreases at a constant rate with time, as is typical in epidermal wound healing [20].

An expression for the rate of change of wound conductance that embodies the above ideas is

$$
\dot{k}=-\frac{\eta k_{d}^{2} p_{a}}{v_{\max }}\left|\frac{k}{k_{d}}\right|^{-1 / n} \frac{k}{k_{d}}\left[1-\left\{\frac{\left(p / p_{a}-1\right)\left|k / k_{d}\right|^{1 / n}}{\delta}\right\}^{2}\right],
$$

where $\eta$ and $\delta$ are dimensionless constants with typical values of 0.01 and 1.0, respectively. For the hydraulic index $n$ we use the value 3 corresponding to a slot [13, page 583] (although we do not thereby intend to imply anything specific about the wound geometry).

Equation (2.3) gives a maximum rate of healing when the knee pressure is equal to the arterial pressure $\left(p=p_{a}\right)$, and a healing rate that vanishes when the wound closes $(k=0)$. Guided by contemporary thoughts [4], it assumes that the wound healing is retarded by the shear stress from blood flow through the arterioles and cancellous bone to the knee cavity.

2.1.3. Summary The simple knee drainage model (2.1)-(2.3) is a coupled firstorder system of ordinary differential equations that can be solved to yield the knee volume $v$ and wound conductance $k$ as functions of time. The model of the recovering knee comprises:

- knee pressure as a monotonically increasing function of knee volume;

- fixed suction pressure;

- fixed arterial (arteriole) pressure;

- fixed hydraulic conductance (ratio of flow to driving pressure difference) from knee to drain; and

- hydraulic conductance from arteries (arterioles) to knee that is a dynamic system parameter.

The artery-to-knee opening heals in time, but at a rate retarded by arterial flow. Sufficient arterial shear can inhibit or actually reverse healing. 


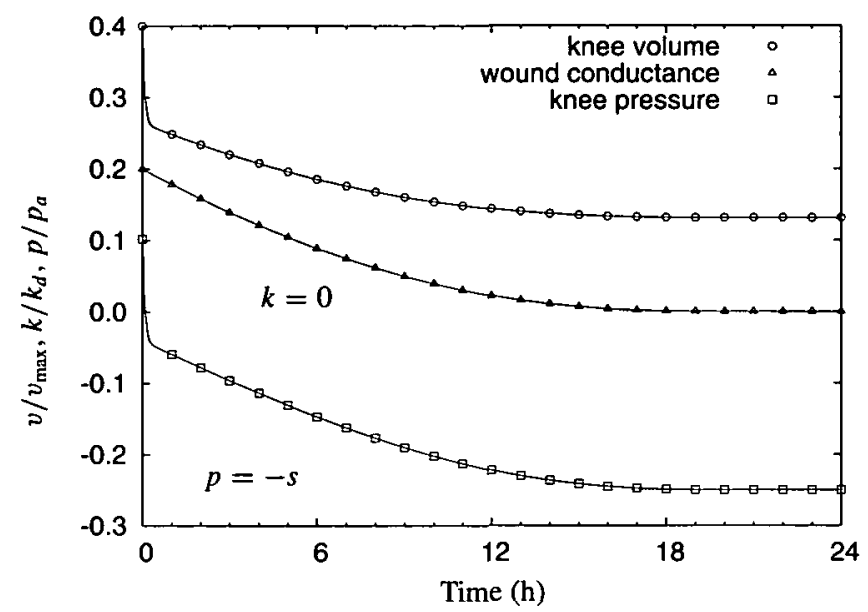

FIGURE 3. Predicted knee volume (normalised by $\left.v_{\max }\right)$, knee pressure $\left(/ p_{a}\right)$, and wound conductance $\left(/ k_{d}\right)$ for $v_{\max }=130 \mathrm{~mL}, p_{a}=40 \mathrm{mmHg}, k_{d}=33 \mathrm{~mL} / \mathrm{h} . \mathrm{mmHg}, s=p_{a} / 4, \alpha=0.5, v_{0}=0.3 v_{\max }$, $\eta=0.01, n=3$, and $\delta=1$. Initial conditions were $v(0)=0.4 v_{\max }$ and $k(0)=0.2 k_{d}$.

2.2. Model solution and results The above equations were solved numerically using the routine LSODE [10] in GNU Octave [7], which adapts time step and order to achieve a specified accuracy (better than graphical accuracy in all plots presented in this paper). An example of the model output is shown in Figure 3. After about $20 \mathrm{~h}$ the system reaches a steady state in which the drainage from the knee ceases and the knee volume equals the value corresponding to the applied suction in (2.2) or Figure 2; that is, $p=-s=-p_{a} / 4=-10 \mathrm{mmHg}$.

2.3. Phase plane analysis The example presented in Figure 3 shows one possible time history of the mathematical model describing the knee damage; to investigate all possible histories we plot the phase plane [15, page 697, for example] of $v$ and $k$, as in Figure 4.

The nullclines

$$
\begin{aligned}
(\dot{k}=0) & k=0 \quad \text { and } \frac{k}{k_{d}}=\left(\frac{\delta}{\left|1-p(v) / p_{a}\right|}\right)^{n} \\
\text { and } \quad(\dot{v}=0) & \frac{k}{k_{d}}=\frac{p(v)+s}{p_{a}-p(v)}
\end{aligned}
$$

are respectively the healing margin (separating states in which $k$ increases from those in which it decreases) and the swelling margin. Here the swelling margin is stable as $\dot{v}<0$ to its right and $\dot{v}>0$ to its left; however, the branch of the healing margin in the first quadrant is unstable as $\dot{k}<0$ below and $\dot{k}>0$ above the curve in Figure 4 . 


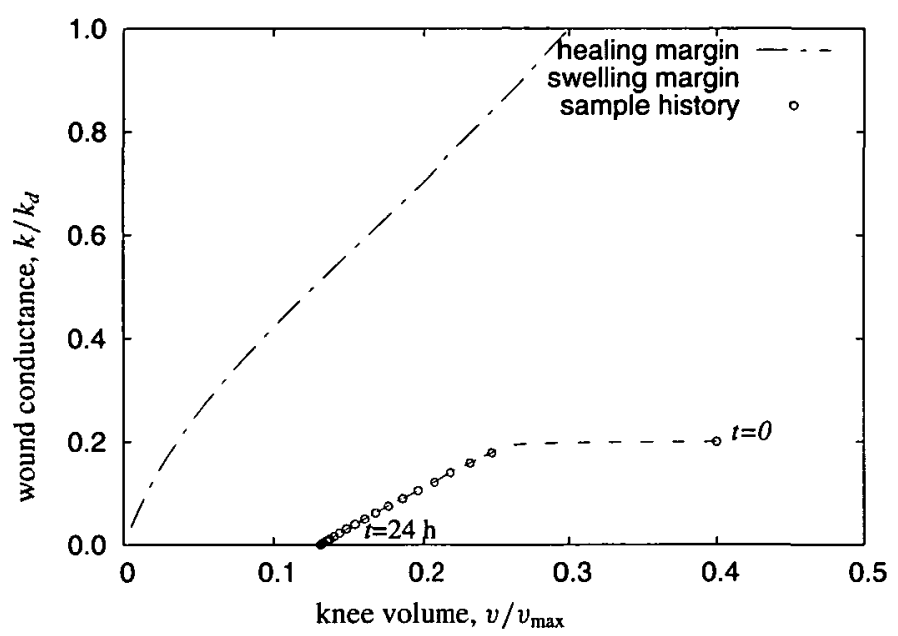

FIGURE 4. Phase plane plot corresponding to the case presented in Figure 3. Also shown are nullclines $\dot{v}=0$ (dotted) and $\dot{k}=0$ (chain). Note that $\dot{k}=0$ on $k=0$ too. The trajectory is the history shown in Figure 3 with points (o) every 30 minutes.

The $k=0$ branch of the healing margin is stable. The system's equilibria occur at the intersections of the nullclines; in this case there is only one, on $k=0$ near $v=0.13 v_{\max }$.

If a higher suction is applied, the swelling margin (2.5) shifts left (meaning that even smaller knee volumes are shrunk) while the healing margin (2.4) is unaffected. Figure 5 shows a case in which the suction is increased to $s=p_{a}=40 \mathrm{mmHg}$ which is sufficient to cause the swelling margin to cross the healing margin and create two new equilibrium points.

From the arrangement and stability of the nullclines (or using linearisation [15, page 700, for example]) we can characterise the three equilibria: the lowest stationary point in Figure 5, like the sole equilibrium in Figure 4, is a stable node; the highest is a stable focus, and the intermediate is a saddle point. The significance of the bifurcation is that although in the history drawn in Figure 5 the knee again proceeds toward the stable node on the line $k=\dot{k}=0$, it may be inferred from the arrangement of the nullclines that if the initial wound was worse (slightly higher $k$ ) then the trajectory would pass above the saddle point and up toward the stable focus. In the first case (regime I) the wound heals normally. In the other (regime II) the high suction draws high bleeding and drainage flow rates through the knee, stressing the wound and preventing its healing.

The model results imply that high suction values can lead to an undesirable situation in which the wound is prevented from healing. This situation can be avoided by using low or negative suction values. The choice of suction level is a compromise between 


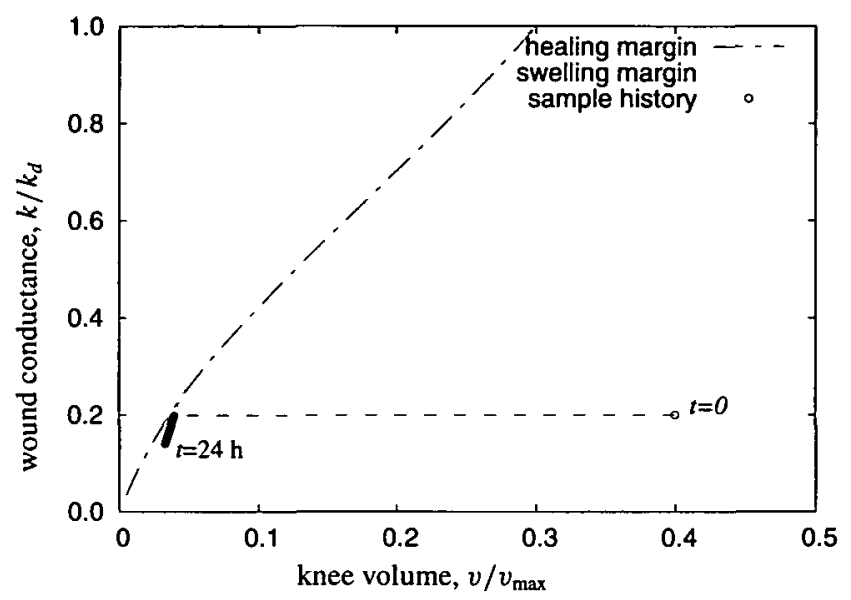

FIGURE 5. Phase plane like Figure 4 but with stronger suction $\left(s=p_{a}\right)$.

excessive knee cavity swelling (insufficient suction) and too much total drainage (excessive suction). Further model runs (not shown) suggest that the most favourable drainage suction is actually slightly negative (that is, pressure towards the knee), around $s=-10 \mathrm{mmHg}$. In this case the overall pressure difference between the knee and drain is enough to promote drainage without excessive swelling, and the model predicts successful healing. On the other hand, suction above a certain value leads to regime II and the model suggests the possibility of retarding healing.

\section{Prospective analysis}

3.1. Methods To test the hypothesis of negative suction drainage suggested by our mathematical model of knee drainage, ten consecutive patients were analysed prospectively between July and November 2000 following primary total knee replacement. The procedure was performed with the use of a tourniquet, and a medial parapatella approach (see, for example, [9]) was used in all patients. Components were implanted with polymethyl-methacrylate cement. Five patients received LCS and five Genesis II prostheses. The tourniquet was released after wound closure and compressive bandage application in all patients. A single Bellovac drain was inserted into the knee joint at the end of the procedure. Enough suction was applied to the drain to allow a column of fluid to reach the bellow only. After this, the drain was kept at a vertical elevation approximately $15 \mathrm{~cm}$ above the knee joint, with no suction applied. Assuming a hydrostatic pressure variation, this elevation difference between the drain and knee results in an applied static back-pressure of about $10 \mathrm{mmHg}$ (that is, $s=-10 \mathrm{mmHg}$ ). 


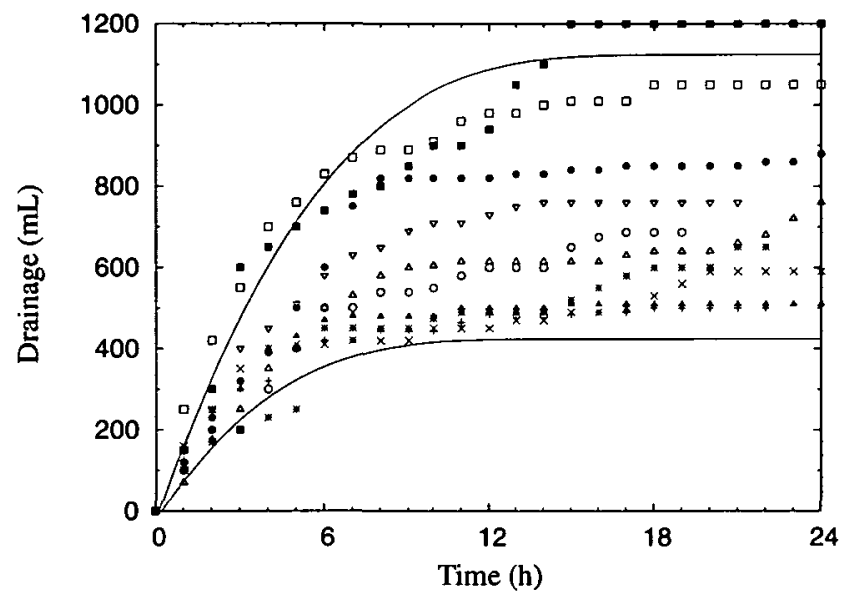

FIGURE 6. Drainage following total knee replacement: point types distinguish patients and the two curves are model results with $k(0)=0.12 k_{d}$ (lower) and $k(0)=0.25 k_{d}$ (upper); other model parameters as in Figures 3-4, except $s=-10 \mathrm{mmHg}$.

Patients began active movement and full-weight bearing on the first postoperative day. Prophylaxis against thromboembolism included: aspirin $150 \mathrm{mg}$ daily for six weeks for two of the patients, enoxaparin $40 \mathrm{mg}$ daily continued for the entire inpatient stay for another two, and no prophylaxis in the remaining six patients. Measurements of drainage were recorded hourly. Patients were followed up for a minimum of three months (average 4.9 months).

3.2. The suction drain The Bellovac drain is an intermittent spring-loaded device that provides a suction that decreases as the bellows relax. Experiments in our laboratory demonstrated that the pressure provided varied from approximately $130 \mathrm{mmHg}$ initially (with bellows fully compressed) to zero when the bellow was relaxed. The bellow volume is approximately $400 \mathrm{~mL}$. Experiments also demonstrated that by devacuuming the device it was subject to atmospheric pressure and could be used as a free drainage system. Under these conditions, the applied suction is solely determined by the vertical elevation of the free surface of the drain relative to the knee (that is, a hydrostatic pressure variation). For example, if the free surface of the drain is $z \mathrm{~mm}$ above the knee, the applied pressure at the knee is $\left(\rho_{b} / \rho_{H_{g}}\right) z \mathrm{mmHg}$, where $\rho_{b}$ is the density of blood, and $\rho_{H g}$ is the density of mercury.

3.3. Results Results of drainage following prospective analysis are shown in Figure 6. Drains averaged $760 \mathrm{~mL}$ (range $500-1200 \mathrm{~mL}$, standard deviation $230 \mathrm{~mL}$ ) in volume and were left in situ for 23 hours (range 19-24 h).

There were no complications noted after a minimum of 3 months follow-up, and 


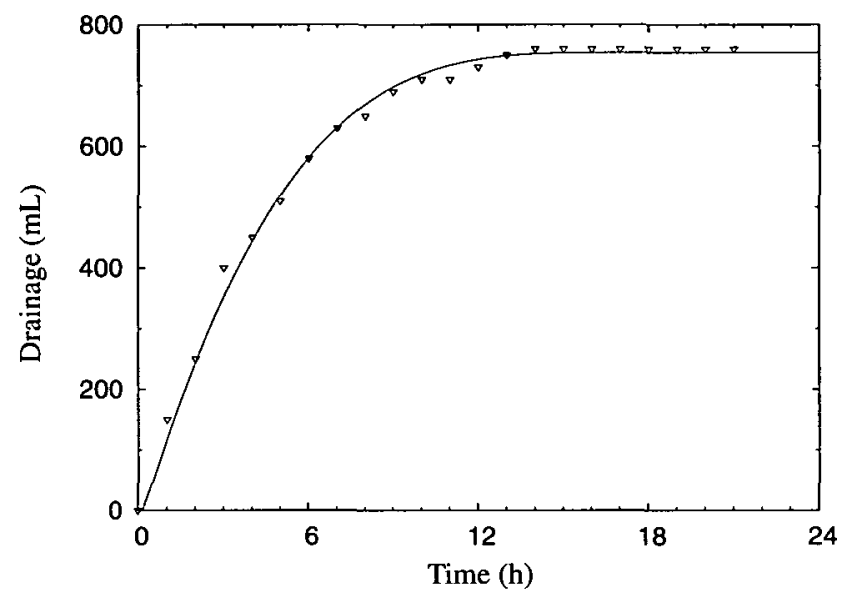

FIGURE 7. Comparison of measured drainage with the predictions of the mathematical model for a single patient. In this case $k(0)=0.184 k_{d}$ was selected to give a good match to the measured data, and all other model parameters are as in Figure 6.

no patient required postoperative transfusion.

\section{Discussion}

4.1. Comparison with mathematical model Prior to the trials the model proved a useful tool to analyse the qualitative behaviour of knee drainage, even though the values of some parameters were uncertain. Here we assess whether the model is capable of reproducing the measured data.

One of the advantages of the trials is that most of the model parameters (including $s$ and $p_{a}$ ) are known or can be deduced. The main unknown parameters are the conductance $k_{d}$ between the knee and the suction drain and the initial conductance $k(0)$ of the path from the arteries to the knee cavity - presumably the latter may vary somewhat from patient to patient depending on the extent of the wound. The initial volume $v(0)$ of the knee does not have a large impact on the results over a wide range, since the knee capsule volume relaxes toward the swelling margin much more quickly than the wound heals.

The drainage rate predicted by the model is calculated using $k_{d}(p+s)$, as in (2.1). After the brief rapid knee capsule volume relaxation, the drainage rate is initially high then decreases with time. The total drainage is obtained by integrating the drainage rate with respect to time.

The measured data suggests that in most cases the bleeding slows or stops after about $14 \mathrm{~h}$. This observation, in conjunction with other known or estimated parame- 


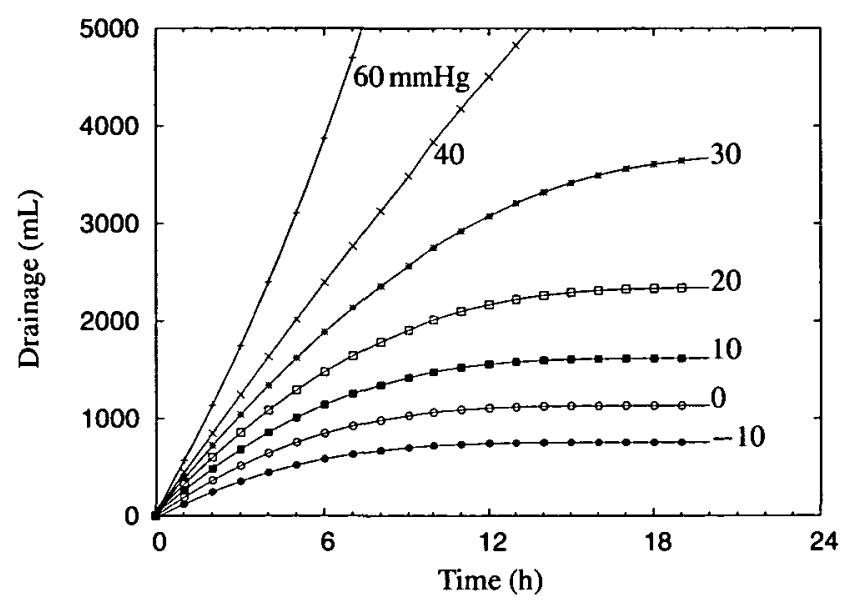

FIGURE 8. Predicted knee drainage for different levels of suction. All model parameters are the same as shown in Figure 7, except for the applied suction which ranges from $-10 \mathrm{mmHg}$ to $60 \mathrm{mmHg}$, as marked.

ters, results in a drain conductance $k_{d}$ of about $33 \mathrm{~mL} / \mathrm{h} . \mathrm{mmHg}$. This value is assumed to be about the same for all patients. The other main unknown is the wound conductance at time zero $k(0)$. It was found that values of $k(0)$ between $0.12 k_{d}$ and $0.25 k_{d}$ produced model predictions which spanned all the measurements, as shown in Figure 6. A particular value of $k(0)$ can yield excellent agreement between the model and the measured drainage; for example, Figure 7 shows a comparison between the measured drainage for an individual patient and the model predictions with $k(0)=0.184 k_{d}$.

The trials were performed with a suction of $s=-10 \mathrm{mmHg}$. Naturally it is of interest to view the model predictions for higher suction values. Figure 8 shows the predicted drainage volumes for suction values ranging from $-10 \mathrm{mmHg}$ to $60 \mathrm{mmHg}$ (holding all other parameters constant). As the suction pressure is increased it can be seen that the total volume of blood drained also increases. For suction values up to $20 \mathrm{mmHg}$ the drainage ceases after about $14 \mathrm{~h}$ (that is, the solution reaches the desired steady state as per regime I), but for large suction values the drainage continues. The latter cases represent the undesirable outcome in which the wound is prevented from healing by the shear stresses (regime II). These predictions support the use of low negative suction values and show that high suction values can result in excessive blood loss.

4.2. Conclusion In reviewing previous total knee replacements at our facility, it was obvious that the suction applied through a compressed Bellovac drain was intermittent, highly variable, and difficult to interpret. The actual amount of suction applied to the 
knee at any one time was dependent on the drainage volume, how often the bellow was recompressed, and the vertical elevation of the drain relative to the knee.

Given this variability-and the uncertainty surrounding the optimal suction to apply - a mathematical model was formulated to assess drainage after total knee replacement. To test the above model, drainage following ten total knee replacements was prospectively analysed utilising the above parameters. Constant suction was applied at a value of approximately $-10 \mathrm{mmHg}$ (that is, pressure towards the knee joint), which was achieved by allowing free drainage and elevating the bellow $15 \mathrm{~cm}$ above the knee. This value corresponded with that suggested by our mathematical drainage model and, as illustrated in the preceding figures, the results parallel those predicted.

These results support our proposal that the postoperative knee should be drained in a constant fashion at a suction much lower than is currently employed by most surgeons. This conclusion is in agreement with previous reports advocating both constant and low-pressure suction.

Two concerns exist, however, with the use of a suction-free system. Firstly, enough suction must be applied to avoid postoperative hæmatoma and the subsequent complications of infection and wound breakdown. No such complication occurred in our prospective group, although the numbers were not sufficient to give this significance. Berman et al. demonstrated increased drainage volumes and improved wound healing following the use of a constant suction device [5]. Regime I of our model demonstrates that at this suction, a steady state is reached, at which the knee volume is static. The second problem with a suction-free drain is the theoretical risk of retrograde migration of bacteria due to the lack of negative pressure. Previous studies demonstrating increased infection rates with suction-free systems used drains left in situ for prolonged periods, or drains that were 'open' to the environment [18]. In our case, the overall pressure gradient is away from the knee, and the system is 'closed', thereby minimising the risk of retrograde migration of bacteria.

A closed drainage system providing a small amount of positive pressure in a constant fashion has been demonstrated to be a valid alternative to the systems most often currently employed. It provides drainage that is more reliable and predictable while safe. A larger prospective trial is warranted to conclusively confirm our analysis.

\section{References}

[1] G. Adalberth, S. Bystrom, K. Kolstad, H. Mallmin and J. Milbrink, "Post-operative drainage of knee arthroplasty is not necessary", Acta Orthop. Scand. 69 (5) (1998) 475-478.

[2] K. J. Beer, A. V. Lombardi, T. H. Mallory and P. K. Vaughn, "The efficacy of suction drains after routine total joint arthroplasty", J. Bone Joint Surg. Am. 73 (1991) 584-587. 
[3] G. Benoni and H. Fredin, "Low- or high-vacuum drains in hip arthroplasty?", Acta Orthop. Scand. 68 (2) (1997) 133-137.

[4] S. A. Berger and L. D. Jou, "Flows in stenotic vessels", Ann. Rev. Fluid Mech. 32 (2000) 347-382.

[5] A. T. Berman, D. Fabiano, S. J. Basacco and A. A. Weiss, "Comparison between intermittent (spring-loaded) and continuous closed suction drainage of orthopedic wounds: A controlled clinical trial", Orthopedics 13 (3) (1990) 309-314.

[6] C. J. Drinkwater and M. J. Neil, "Optimal timing of wound drain removal following total joint arthroplasty", J. Arthroplasty 10 (2) (1995) 185-189.

[7] J. W. Eaton, GNU Octave Manual (Network Theory, 2002).

[8] P. Geborek, U. Moritz and F. A. Wollheim, "Joint capsular stiffness in knee arthritis. relationship to intraarticular volume, hydrostatic pressures and extensor muscle function", J. Rheumatol. 16 (1989) 1351-1358.

[9] P. Gopinath and Arun B., "Surgical exposures for primary total knee replacement", J. Orthop. 1 (1) (2004) e6.

[10] A. C. Hindmarsh, "ODEPACK, a systematized collection of ODE solvers", in Scientific Computing (ed. R. S. Stepleman), (North-Holland, Amsterdam, 1983), 55-64.

[11] B. J. Holt, N. L. Parks, G. A. Engh and J. M. Lawrence, "Comparison of closed-suction drainage and no drainage after primary total knee arthroplasty", Orthopedics 20 (12) (1997) 1121-1125.

[12] Y. H. Kim, S. H. Cho and R. S. Kim, "Drainage versus nondrainage in simultaneous bilateral total knee arthroplastics”, Clin. Orthop. 347 (1998) 188-193.

[13] H. Lamb, Hydrodynamics, 6th ed. (Cambridge University Press, Cambridge, 1932).

[14] P. B. Loder, G. H. Smith, S. Morris, C. P. Bambach and R. C. Smith, "A randomised comparison of three drainage systems following cholecystectomy", Aust. N. Z. J. Surg. 57 (1987) 531-535.

[15] J. D. Murray, Mathematical Biology (Springer, Berlin, 1989).

[16] D. Ovadia, E. Luger, J. Bickels, A. Menachem and S. Dekel, "Efficacy of closed wound drainage after total joint arthroplasty", J. Arthroplasty 12 (3) (1997) 317-321.

[17] M. J. Parker, C. P. Roberts and D. Hay, "Closed suction drainage for hip and knee arthroplasty", $J$. Bone Joint Surg. Am. 86 (6) (2004) 1146-1152.

[18] H. J. Raves, M. Slifkin and D. L. Diamond, "A bacteriologic study comparing closed suction and simple conduit drainage", Am. J. Surg. 148 (1984) 618-620.

[19] M. A. Ritter, E. M. Keating and P. M. Faris, "Closed wound drainage in total hip or total knee replacement", J. Bone Joint Surg. Am. 76 (1994) 35-38.

[20] J. M. Snowden, "Wound contraction: A quantitative interpretation", Aust. J. Exp. Biol. Med. Sci. 59 (2) (1981) 203-217.

[21] A. Sperber and T. Wredmark, "Tensile properties of the knee joint capsule at elevated intra-articular pressure", Acta Orthop. Scand. 69 (5) (1998) 484-488.

[22] P. Zamora-Navas, F. Collada-Torres and F. de la Torre-Solis, "Closed suction drainage after total knee arthroplasty", Acta Orthopadica Belgica 65 (1) (1999) 44-47. 